\title{
Epidemiology and outcomes of pediatric intracranial aneurysms: comparison with an adult population in a 30-year, prospective database
}

\author{
Risheng Xu, MD, PhD, ${ }^{1}$ Michael E. Xie, AM, ${ }^{1}$ Wuyang Yang, MD, ${ }^{1}$ Philippe Gailloud, MD, ${ }^{2}$ \\ Justin M. Caplan, MD, ${ }^{1}$ Christopher M. Jackson, MD, ${ }^{1}$ Eric M. Jackson, MD, ${ }^{1}$ Mari L. Groves, MD, ${ }^{1}$ \\ Shenandoah Robinson, MD, ${ }^{1}$ Alan R. Cohen, MD, ${ }^{1}$ Judy Huang, MD, ${ }^{1}$ and Rafael J. Tamargo, MD ${ }^{1}$ \\ 1Department of Neurosurgery, Johns Hopkins University School of Medicine, Baltimore, Maryland; and 2Division of Interventional \\ Neuroradiology, Johns Hopkins University School of Medicine, Baltimore, Maryland
}

\begin{abstract}
OBJECTIVE Pediatric intracranial aneurysms are rare. Most large series in the last 15 years reported on an average of only 39 patients. The authors sought to report their institutional experience with pediatric intracranial aneurysms from 1991 to 2021 and to compare pediatric patient and aneurysm characteristics with those of a contemporaneous adult cohort.
\end{abstract}

METHODS Pediatric ( $\leq 18$ years of age) and adult patients with one or more intracranial aneurysms were identified in a prospective database. Standard epidemiological features and outcomes of each pediatric patient were retrospectively recorded. These results were compared with those of adult aneurysm patients managed at a single institution over the same time period.

RESULTS From a total of 4500 patients with 5150 intracranial aneurysms admitted over 30 years, there were 47 children with 53 aneurysms and 4453 adults with 5097 aneurysms; $53.2 \%$ of children and $36.4 \%$ of adults presented with a subarachnoid hemorrhage (SAH). Pediatric aneurysms were significantly more common in males, more likely giant $(\geq 25$ $\mathrm{mm}$ ), and most frequently located in the middle cerebral artery. Overall, $85.1 \%$ of the pediatric patients had a modified Rankin Scale score $\leq 2$ at the last follow-up (with a mean follow-up of 65.9 months), and the pediatric mortality rate was $10.6 \%$; all 5 patients who died had an SAH. The recurrence rate of treated aneurysms was $6.7 \%(1 / 15)$ in the endovascular group but $0 \%(0 / 31)$ in the microsurgical group. No de novo aneurysms occurred in children (mean follow-up 5.5 years). CONCLUSIONS Pediatric intracranial aneurysms are significantly different from adult aneurysms in terms of sex, presentation, location, size, and outcomes. Future prospective studies will better characterize long-term aneurysm recurrence, rebleeds, and de novo aneurysm occurrences. The authors currently favor microsurgical over endovascular treatment for pediatric aneurysms.

https://thejns.org/doi/abs/10.3171/2021.6.PEDS21268

KEYWORDS pediatric; intracranial aneurysm; cerebral aneurysms; microsurgery; endovascular; vascular disorders

$\mathrm{P}$ EDIATRIC intracranial aneurysms are a rare clinical entity whose epidemiology is coming into focus based on the cumulative experience at large neurosurgical centers. ${ }^{1,2}$ To date, most reports of pediatric aneurysms consist of case reports and small case series. ${ }^{3-20}$ Most large series in the last 15 years reported on an average of 39 patients. We previously presented a series of 19 patients treated at our institution from 1991 to 2004 over the course of 13 years. ${ }^{21}$ Herein, we report our updated institutional experience from 1991 to 2021, over 30 years, and compare pediatric patient and aneurysm character- istics with those of adults with intracranial aneurysms treated at our institution over the same time period. We describe the angiographic and neurological long-term outcomes of these patients, including outcomes of endovascular treatments versus microsurgery.

\section{Methods}

Pediatric patients ( $\leq 18$ years of age) who presented with an intracranial aneurysm were identified in a prospectively managed cerebrovascular database. This data-

ABBREVIATIONS ACA = anterior cerebral artery; $\mathrm{AVM}=$ arteriovenous malformation; $E V D$ = external ventricular drain; ICA = internal carotid artery; ICH = intracerebral hemorrhage; $\mathrm{IVH}=$ intraventricular hemorrhage; $\mathrm{MCA}=$ middle cerebral artery; $\mathrm{mRS}=$ modified Rankin Scale; $\mathrm{PCA}=$ posterior cerebral artery; $\mathrm{SAH}=$ subarachnoid hemorrhage. SUBMITTED May 19, 2021. ACCEPTED June 10, 2021.

INCLUDE WHEN CITING Published online September 10, 2021; DOI: 10.3171/2021.6.PEDS21268. 

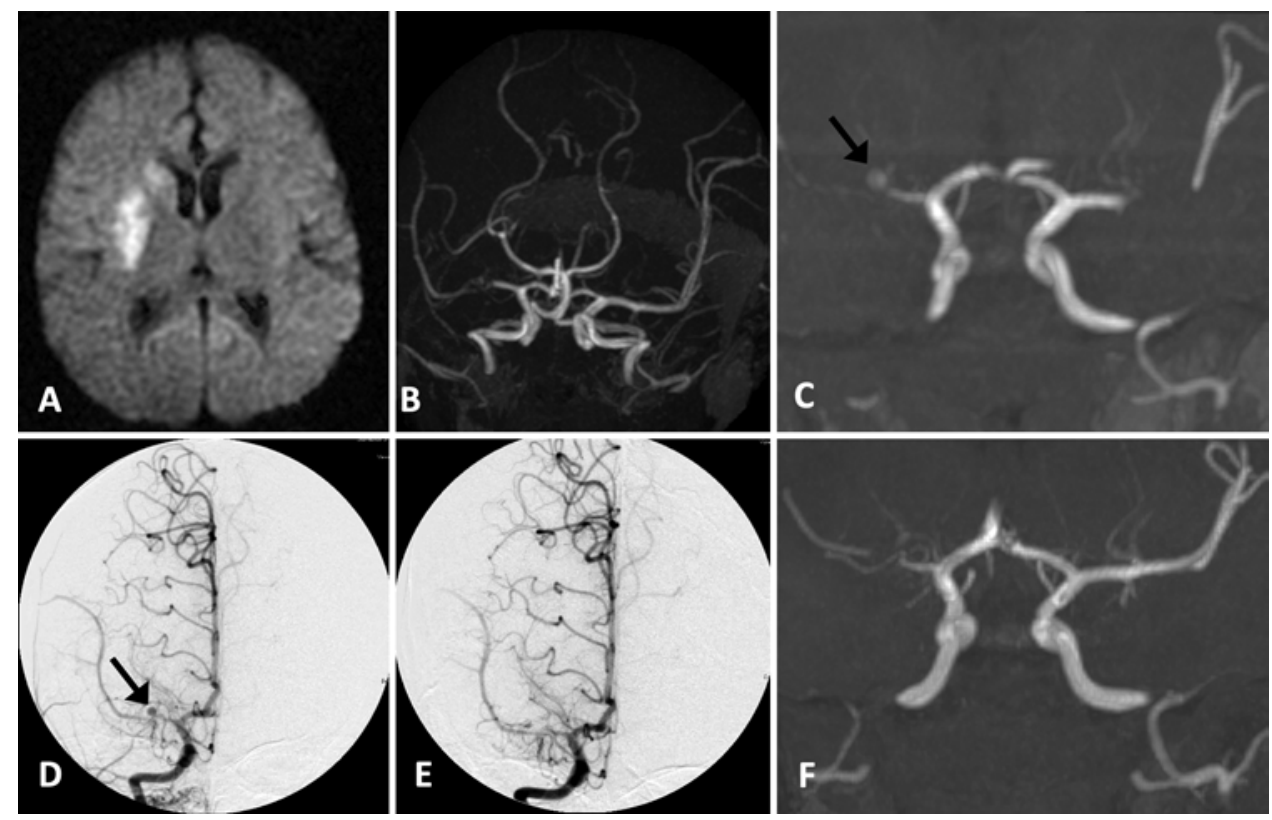

FIG. 1. A 6-month-old female patient presented in 2006 with seizures in the setting of an upper respiratory tract infection and was found to have a right MCA stroke. The patient underwent an extensive stroke and hypercoagulable workup, which was notable only for a reduced protein $\mathrm{C}$ activity level, and homozygosity for the MTHFR polymorphism C66T. A and B: Axial diffusion-weighted $M R$ image $(A)$ revealing the right MCA stroke affecting primarily the basal ganglia, and an MR angiogram (B) showing that no cerebral aneurysm was seen at that time. The patient was placed on a daily aspirin regimen. C: A routine 1-year follow-up MR angiogram demonstrating a right $\mathrm{MCA} \mathrm{M}_{1}$ aneurysm (arrow). D: A cerebral angiogram obtained at 18 months (delayed due to scheduling issues and viral illnesses) confirmed the aneurysm (arrow). The case was discussed in the neurovascular conference, and, given the patient's need for daily aspirin as well as retrograde filling of the MCA via pial collaterals from the ACA territory, it was decided that endovascular treatment would be more favorable. E: Angiogram showing that the patient underwent successful coiling; she was discharged the next day. F: MR angiogram showing no recurrent aneurysm. The patient continued to do well at the last follow-up (140 months) and was able to play the piano.

base is maintained under IRB approval. A total of 4500 patients with 5150 aneurysms were treated over the course of 30 years at the Johns Hopkins Hospital and the Johns Hopkins Bayview Medical Center, including 47 pediatric patients with 53 aneurysms.

Radiographic studies, operative reports, and hospital records were retrospectively reviewed for each patient. The age, sex, and clinical presentation of patients were noted, and the morphology, size, and location of each aneurysm were documented. Medical comorbidities were also recorded. We present our overall institutional experience from 1991 to 2021, and we provide a subgroup summary of patients treated since our last publication in 2005.

A diagnosis of subarachnoid hemorrhage ( $\mathrm{SAH}$ ) was confirmed with a head CT or with analysis of CSF obtained by lumbar puncture. As an institution, we have been early adopters of endovascular technologies since the early 1990s. Thus, four-vessel angiography was performed within 24 hours of admission unless an adequate angiogram was available from the referring institution. Patients were monitored in the pediatric intensive care unit while awaiting definitive treatment and received nimodipine, phenytoin or levetiracetam, intravenous fluids, and aggressive blood pressure monitoring to avoid hypertension. Patients who presented with an SAH were usually treated within 24 hours of hemorrhage.

Pediatric aneurysm patients at our institution are generally referred to both the neurosurgery and neurointerven- tional radiology services. Management of the aneurysms is discussed at a multidisciplinary meeting encompassing both open vascular and endovascular providers. The treatment for each aneurysm via open clipping or wrapping versus endovascular coiling, flow diversion, or vessel sacrifice was recorded. Representative cases are summarized in Figs. 1 and 2. For clipping of cerebral aneurysms, intraoperative EEG and somatosensory evoked potential monitoring were routinely performed, and either intraoperative or postoperative angiography was performed. Follow-up evaluation was completed by the treating neurosurgeon and/or the pediatric neurologist during outpatient clinic visits. Neurological functional outcomes were assessed using the modified Rankin Scale (mRS). In general, patients were monitored with MRI, MRA, or cerebral angiography starting 5 years after surgery. Residual aneurysms were monitored for stability, and any new aneurysms were noted.

\section{Results}

Over 30 years, from 1991 to 2021, 4500 patients with 5150 aneurysms were admitted to our institution. Of these, there were 47 pediatric patients with 53 aneurysms and 4453 adults with 5097 aneurysms. Of the 47 pediatric patients with intracranial aneurysms (Tables 1 and 2), 3 $(6.4 \%)$ were $\leq 1$ year of age, $16(34.0 \%)$ were between the ages of 1 and 10 years, and $28(59.6 \%)$ were between 10 

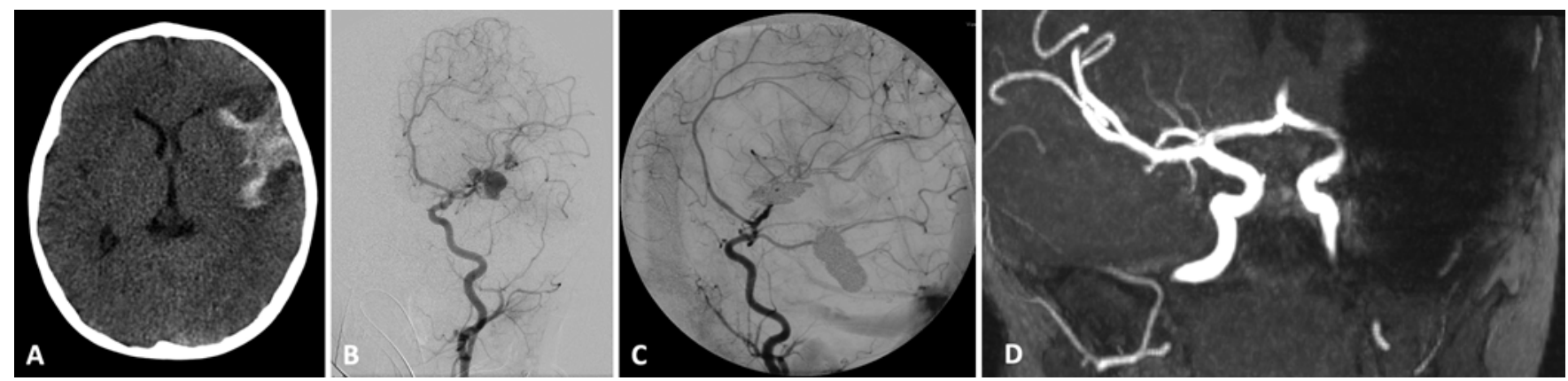

FIG. 2. A 12-month-old male patient presented with lethargy, vomiting, diarrhea, and seizures in 2009. A: Head CT scan demonstrating a left SAH centered around the left sylvian fissure. B: Cerebral angiogram demonstrating $2 \mathrm{MCA}$ aneurysms that were both irregular. The case was presented at the neurovascular conference, and the decision was made for surgical clipping given the morphology and multiplicity of the aneurysms. C: Intraoperative angiogram demonstrating successful obliteration of all aneurysms after the patient had undergone an uneventful clipping. He was discharged to rehabilitation after weaning of the EVD. D: MR angiogram obtained at the last follow-up, in 2016, when the patient was attending school and able to ambulate independently with only mild speech delay.

and 18 years of age; 29 patients $(61.7 \%)$ were male, giving a male-to-female ratio of 1.6:1. Compared with our prospective cohort of 4453 adult patients treated for aneurysms at our institution during this period, of whom 3429 (77.0\%) were female, pediatric patients had a statistically higher likelihood of being male $(\mathrm{p}<0.001)$ (Table 3$)$.

Out of 47 pediatric patients, $25(53.2 \%)$ presented with an SAH, which was statistically higher compared with the adult patient population $(36.4 \%, \mathrm{p}=0.026)$. Of those 25 patients, $19(76 \%)$ presented with a Hunt and Hess grade of I to III, and 6 patients (24\%) presented with a grade IV or V SAH. Two of the 3 patients with a grade V SAH presented with no hydrocephalus but with global anoxic injury with loss of gray-white matter differentiation. Autopsies for these 2 patients found no additional cause of death other than SAH. The other patient with a grade V SAH had a massive intracerebral hemorrhage (ICH) with an intraventricular hemorrhage (IVH) and a midline shift with hydrocephalus. The family declined CSF diversion and surgical intervention and declined autopsy. Of the pediatric patients who did not present with an SAH, 10 (46\%) presented with neurological symptoms such as cranial nerve palsies, hemiparesis, seizures, or nausea/vomiting, and 12 patients $(55 \%)$ presented with aneurysms found incidentally during workup of other etiologies such as headache or trauma. In total, 17 pediatric patients $(36.2 \%)$ in our series presented with evidence of hydrocephalus. One patient with a Spetzler-Martin grade V AVM had an intranidal aneurysm. Two patients had associated brain tumors; one patient had a desmoplastic infantile ganglioma, and the other had an anaplastic astrocytoma. One patient had a previous occult stroke, and 1 patient had a Chiari II malformation with an associated myelomeningocele and shunt. Three patients had a history of hematological disorders; 2 had sickle cell disease, and 1 had concomitant thalassemia. One patient had a G6PD (glucose-6-phosphate dehydrogenase) deficiency, 1 had a history of nephrotic syndrome, and 1 had Stargardt disease. These comorbidities were not thought to be related to the aneurysms. The patient with a history of nephrotic syndrome had normal kidney function and no evidence of proteinuria at the time of surgery. Three patients were seen by our medical genetics team for evaluation of connective tissue disorders, of whom 2 patients were found to be negative; the other patient was found to have a variant of unknown significance in the COL5A1 gene. One patient presented with a mycotic aneurysm due to periodontal infection.

Of 53 pediatric intracranial aneurysms, $39(73.6 \%)$ were located in the anterior circulation, and $14(26.4 \%)$ were located in the posterior circulation. Of note, posterior communicating artery aneurysms were classified as posterior circulation aneurysms, in line with seminal natural history studies such as International Study of Unruptured Intracranial Aneurysms (ISUIA) I and ISUIA II..$^{22}$ Although the overall proportion of anterior and posterior aneurysms was similar when compared with the adult population of intracranial aneurysms $(p=0.771)$, the distribution of aneurysms specifically at each location differed significantly $(\mathrm{p}<0.001)$ (Table 3). Within the anterior circulation, pediatric patients were more likely to have aneurysms within the cavernous internal carotid artery (ICA), ICA termination, and the distal anterior cerebral artery (ACA). However, they were less likely to have aneurysms within the anterior communicating artery complex compared with their adult counterparts. Within the posterior circulation, pediatric patients were more likely to have vertebral artery and posterior cerebral artery (PCA) aneurysms. Ten of 53 $(18.9 \%)$ pediatric aneurysms were giant, compared with 119 of $5097(2.3 \%)$ aneurysms in adults $(\mathrm{p}<0.001)$.

Although 53 pediatric intracranial aneurysms were encountered, 47 aneurysms were treated, as 3 pediatric patients arrived with Hunt and Hess grade V SAH, and their neurological condition deteriorated quickly to brain death despite maximum medical measures. Two patients who presented with an SAH had multiple aneurysms, but both patients died before their unruptured aneurysms could be addressed. Fifteen aneurysms were treated with endovascular techniques, which included coiling, balloon occlusion, and/or flow diversion. Endovascular embolization was attempted in a distal lenticulostriate artery aneurysm, but stable access could not be reached, and the aneurysm thrombosed spontaneously on follow-up imaging. Thirty 
TABLE 1. Patient characteristics of all pediatric intracranial aneurysms, 1991-2021

\begin{tabular}{|c|c|c|c|c|c|c|c|c|c|c|c|}
\hline $\begin{array}{l}\text { Pt } \\
\text { No. }\end{array}$ & $\begin{array}{l}\text { Age } \\
\text { (yrs) }\end{array}$ & Sex & Location & Type & Presentation & Comorbidity & Intervention & $\begin{array}{c}\text { FU } \\
(\mathrm{mos})\end{array}$ & $\begin{array}{l}\text { mRS } \\
\text { on FU }\end{array}$ & $\begin{array}{l}\text { Residual } \\
\text { Anr }\end{array}$ & $\begin{array}{c}\text { Growing } \\
\text { Anr }\end{array}$ \\
\hline 1 & 0.1 & $\mathrm{~F}$ & Lt cavernous & S & $\begin{array}{l}\text { Bulging eye, } \\
\text { vomiting }\end{array}$ & None & $\begin{array}{l}\text { Glue \& coil } \\
\text { occlusion }\end{array}$ & 184.9 & 0 & 0 & 0 \\
\hline \multirow[t]{2}{*}{2} & 0.8 & $\mathrm{~F}$ & Lt MCA pseudo & Pseudo & $\begin{array}{l}\text { Hemiparesis, } \\
\text { seizures }\end{array}$ & $\begin{array}{c}\text { Lt desmoplastic infantile } \\
\text { ganglioma resection w/ } \\
\text { CVA, ICH }\end{array}$ & Coil & 13.8 & 2 & 1 & 1 \\
\hline & 0.8 & $\mathrm{~F}$ & Lt MCA pseudo & Pseudo & $\begin{array}{l}\text { Routine } \\
\text { monitoring }\end{array}$ & As above & $\begin{array}{l}\text { Re-coil w/ } \\
\text { flow diversion }\end{array}$ & 13.8 & 2 & 1 & 0 \\
\hline 3 & 1 & M & L MCA $\times 2$ & $S$ & $\mathrm{SAH}$ & None & Clip & 93 & 1 & 0 & 0 \\
\hline 4 & 1.8 & $\mathrm{~F}$ & Rt MCA & S & Rt MCA stroke & $\begin{array}{l}\text { Protein C deficiency, } \\
\text { MTHFR mutation }\end{array}$ & Coil & 140.8 & 1 & 0 & 0 \\
\hline 5 & 1.9 & $\mathrm{~F}$ & Lt cavernous & G & $\begin{array}{l}\text { Bulge in subman- } \\
\text { dibular region }\end{array}$ & Mastoiditis & $\mathrm{BO}$ & 0.7 & 0 & 0 & 0 \\
\hline \multirow[t]{2}{*}{6} & 4.1 & M & $\begin{array}{c}\text { Rt ICA, It ICA, } \\
\text { rt A2 }\end{array}$ & G & SAH & $\begin{array}{l}\text { Hx Hunterian ligation of BA } \\
\text { anr, HC w/ shunt, STAT1 } \\
\text { deficiency, developmental } \\
\text { delay, polyendocrinopathy }\end{array}$ & $\begin{array}{c}\text { Flow diversion } \\
\text { (rt ICA) }\end{array}$ & 1.5 & 6 & 1 & 1 \\
\hline & 4.1 & M & $\begin{array}{c}\text { Rt ICA, It ICA, } \\
\text { rt A2 }\end{array}$ & S & SAH & As above & Coil (It ICA) & 1.5 & 6 & 1 & 0 \\
\hline 7 & 4.5 & M & Rt A2 & $\begin{array}{c}\text { Intranidal } \\
\text { artery }\end{array}$ & $\begin{array}{l}\text { Lt hemiparesis, } \\
\text { numbness }\end{array}$ & Rt S-M grade V AVM & $\begin{array}{l}\text { Glue emboli- } \\
\text { zation }\end{array}$ & 113.3 & 2 & 1 & 0 \\
\hline 8 & 5.3 & M & Lt lenticulostriate & S & SAH & Nephrotic syndrome & Attempted coil & 25.7 & 1 & 0 & 0 \\
\hline 9 & 5.6 & M & Rt PChA & Fusi & $\mathrm{SAH}$ & SC anemia & Clip & 227 & 1 & 0 & 0 \\
\hline 10 & 6.7 & $\mathrm{~F}$ & Lt PCA & G & $\mathrm{SAH}$ & None & None & & 6 & & \\
\hline 11 & 7.5 & M & Lt A1 fusi & G & $\mathrm{HA}$, field cut & None & Clip & 201.8 & 0 & 0 & 0 \\
\hline 12 & 7.8 & M & Lt cavernous & G & $\begin{array}{l}\mathrm{HA}, 3 \text { rd \& 6th } \\
\text { nerve palsies }\end{array}$ & None & BO & 207 & 0 & 0 & 0 \\
\hline 13 & 8.2 & $\mathrm{~F}$ & Lt ICA term & $S$ & SAH & None & Clip & 18.5 & 1 & 0 & 0 \\
\hline 14 & 8.3 & $\mathrm{~F}$ & Lt VA & G & $\mathrm{HA}$ & None & Coil occlusion & 49.9 & 0 & 0 & 0 \\
\hline 15 & 8.3 & M & Lt VA & Fusi & $\mathrm{SAH}$ & None & None & & 6 & & \\
\hline 16 & 8.7 & M & Lt ACA & $S$ & Meningitis & Old stroke & Clip & 13.8 & 1 & 0 & 0 \\
\hline 17 & 9.3 & $\mathrm{~F}$ & $\mathrm{BA}$ & $S$ & SAH & None & Clip & 1 & 0 & 0 & 0 \\
\hline 18 & 9.8 & $\mathrm{~F}$ & Rt ACA & S & Incidental & $\begin{array}{c}\text { Myelomeningocele, Chiari } \\
\text { II, shunted HC }\end{array}$ & Clip & 45.4 & 4 & 0 & 0 \\
\hline 19 & 9.8 & M & $\begin{array}{l}\text { Rt P1 } \\
\text { thalamoperf }\end{array}$ & $\begin{array}{l}\text { Broad- } \\
\text { based }\end{array}$ & Incidental & None & Wrap & 195.8 & 1 & 1 & 0 \\
\hline 20 & 11 & M & Rt ACoA & S & $\mathrm{SAH}$ & Hypertension & Clip & 1.4 & 0 & 0 & 0 \\
\hline 21 & 12 & M & Lt ACA & $S$ & $\mathrm{SAH}$ & None & Clip & 68.8 & 0 & 0 & 0 \\
\hline 22 & 12.4 & M & Rt A1 & Fusi & Incidental & Tuberous sclerosis & Clip & 198.8 & 0 & 0 & 0 \\
\hline 23 & 12.7 & $\mathrm{~F}$ & Rt ICA term & $S$ & SAH & None & Clip & 14 & 0 & 0 & 0 \\
\hline 24 & 12.9 & M & Lt PCoA & S & $\mathrm{SAH}$ & Stargardt, asthma & Clip & 3.1 & 1 & 0 & 0 \\
\hline 25 & 13.2 & M & Rt ACoA & S & $\mathrm{SAH}$ & G6PD deficiency & Clip & 23.2 & 0 & 0 & 0 \\
\hline 26 & 13.2 & M & Lt ICA term & $S$ & $\mathrm{SAH}$ & Tourette's syndrome & Clip & 62.6 & 0 & 0 & 0 \\
\hline 27 & 14 & $\mathrm{~F}$ & Lt PCA & G & $\mathrm{SAH}$ & None & Clip & 135.8 & 0 & 0 & 0 \\
\hline 28 & 14.2 & $\mathrm{~F}$ & Lt MCA & G & $\mathrm{SAH}$ & None & Clip & 125.9 & 3 & 0 & 0 \\
\hline 29 & 14.7 & M & Lt PICA, It MCA & S & $\mathrm{SAH}$ & None & Clip & 42.9 & 0 & 0 & 0 \\
\hline 30 & 14.8 & M & Rt VA, It MCA & Fusi & SAH & AA, IBS & $\begin{array}{l}\text { Coil occlusion } \\
\text { of rt VA anr }\end{array}$ & 3.6 & 6 & 0 & 0 \\
\hline 31 & 14.9 & $\mathrm{~F}$ & $\begin{array}{l}\text { Lt PCoA, It } \\
\text { dorsal ICA }\end{array}$ & S & SAH & SSD & Clip, both anrs & 9.7 & 0 & 0 & 0 \\
\hline 32 & 15.2 & M & Lt cavernous & $S$ & Incidental & Benign hypotonia, migraines & Coil occlusion & 25.2 & 0 & 0 & 0 \\
\hline
\end{tabular}


TABLE 1. Patient characteristics of all pediatric intracranial aneurysms, 1991-2021

\begin{tabular}{|c|c|c|c|c|c|c|c|c|c|c|c|}
\hline $\begin{array}{l}\mathrm{Pt} \\
\text { No. }\end{array}$ & $\begin{array}{l}\text { Age } \\
\text { (yrs) }\end{array}$ & Sex & Location & Type & Presentation & Comorbidity & Intervention & $\begin{array}{c}\text { FU } \\
\text { (mos) }\end{array}$ & $\begin{array}{l}\mathrm{mRS} \\
\text { on FU }\end{array}$ & $\begin{array}{c}\text { Residual } \\
\text { Anr }\end{array}$ & $\begin{array}{c}\text { Growing } \\
\text { Anr }\end{array}$ \\
\hline 33 & 15.4 & M & Lt MCA & S & Incidental & Tonsil surgery & Clip & 18.2 & 0 & 0 & 0 \\
\hline 34 & 15.4 & $M$ & Rt ICA term & $S$ & SAH & None & Clip & 130.4 & 1 & 0 & 0 \\
\hline 35 & 15.8 & $\mathrm{~F}$ & Lt OA & $S$ & Incidental & SSD & Clip & 15.2 & 0 & 0 & 0 \\
\hline 36 & 16.1 & M & Lt ACA & $S$ & SAH & Gum disease/infection & Antibiotics & 0.7 & 0 & 0 & 0 \\
\hline 37 & 16.4 & $\mathrm{~F}$ & Lt PCoA & $S$ & 3rd nerve palsy & None & Clip & 1.5 & 0 & 0 & 0 \\
\hline 38 & 16.5 & M & Lt cavernous & G & $\mathrm{HA}$ & None & $\mathrm{BO}$ & 24.1 & 0 & 0 & 0 \\
\hline 39 & 16.8 & M & Lt SCA & S & Incidental & Head injury during hockey & Clip & 58.3 & 0 & 0 & 0 \\
\hline 40 & 17 & $\mathrm{~F}$ & Lt MCA & S & SAH & None & Clip & 112.6 & 0 & 0 & 0 \\
\hline 41 & 17.3 & M & Lt MCA & G & Lt MCA stroke & None & Clip & 35.6 & 2 & 0 & 0 \\
\hline 42 & 17.5 & M & Rt ICA term & $S$ & SAH & Thalassemia & Clip & 0.5 & 0 & 0 & 0 \\
\hline 43 & 17.5 & M & Rt ICA term & $S$ & $\mathrm{SAH}$ & Migraines & None & & 6 & & \\
\hline 44 & 18.1 & M & Lt ACA & $S$ & Incidental & $\begin{array}{c}\text { ADHD, ruptured ear drum } \\
\text { due to trauma }\end{array}$ & Clip & 97.5 & 0 & 0 & 0 \\
\hline 45 & 18.1 & $\mathrm{~F}$ & BA & S & Incidental & $\mathrm{HA}$ & $\begin{array}{l}\text { Coil w/ flow } \\
\text { diversion }\end{array}$ & 0.2 & 0 & 0 & 0 \\
\hline 46 & 18.2 & $\mathrm{~F}$ & Lt OA & S & Incidental & Asthma, HA & Clip & 35.5 & 0 & 0 & 0 \\
\hline 47 & 18.2 & M & Lt ACoA & $S$ & $\mathrm{SAH}$ & None & Clip & 236.6 & 1 & 0 & 0 \\
\hline
\end{tabular}

$\mathrm{AA}=$ anaplastic astrocytoma; $\mathrm{ACOA}=$ anterior communicating artery; $\mathrm{ADHD}=$ attention-deficit hyperactivity disorder; anr = aneurysm; $\mathrm{BA}=\mathrm{basilar}$ artery; $\mathrm{BO}=$ balloon occlusion; FU = follow-up; fusi = fusiform; $\mathrm{G}=$ giant; $\mathrm{HA}=$ headache; $\mathrm{HC}=$ hydrocephalus; $\mathrm{Hx}=$ history of; IBS = irritable bowel syndrome; OA = ophthalmic artery; $\mathrm{PChA}=$ posterior choroidal artery; $\mathrm{PCoA}=$ posterior communicating artery; pseudo = pseudoaneurysm; $p t=p a t i e n t ; S=$ saccular; $\mathrm{SC}=$ sickle cell; $\mathrm{SCA}=$ superior cerebellar artery; S-M = Spetzler-Martin; SSD = SC disease; term = termination; thalamoperf = thalamoperforator; VA = vertebral artery.

aneurysms received clipping. One aneurysm was discovered intraoperatively to be a broad-based aneurysm with a $\mathrm{P}_{1}$ thalamoperforator emerging from the dome and was deemed too high risk to clip reconstruct and, therefore, was wrapped. One patient with an infectious mycotic aneurysm underwent antibiotic treatment.

Three patients experienced Hunt and Hess grade V SAHs and were neurologically unstable, which progressed to brain death shortly after arriving to the hospital despite maximum resuscitative measures without neurosurgical intervention. Of the 44 treated pediatric patients, 26 (59\%) had at least 2 years of follow-up, 7 (16\%) had between 1 and 2 years of follow-up, and $11(25 \%)$ had $<1$ year of follow-up. Overall, 40 pediatric patients $(85.1 \%)$ had excellent neurological outcomes with an $\mathrm{mRS}$ score $\leq 2$ over a mean follow-up period of 65.9 months. Of the 4 patients with $\mathrm{mRS}$ scores $>2,1$ patient was a 4-year-old male with a history of developmental delay, immune deficiency, and polyendocrinopathy who had a history of a ruptured basilar artery aneurysm that was treated with Hunterian ligation at an outside institution in 2010. In 2011, 1.5 years after his surgery, he was found to have growing bilateral, giant ICA termination aneurysms and was referred to us for treatment. The patient underwent flow diversion of the right ICA aneurysm. Five days after surgery, he experienced a seizure, and head CT demonstrated new ICH and SAH on the left. He underwent coil embolization of the left ICA aneurysm. Despite these measures, he had a worsening left ICH with a massive midline shift and hydrocephalus requiring external ventriculostomy placement and a decompressive hemicraniectomy the following day. Despite maximal medical management, the patient's neurological examination remained poor, and the family elected to withdraw care approximately 3 weeks later. Another patient was a 14-year-old male with a history of a posterior fossa anaplastic astrocytoma who presented with an SAH and hydrocephalus more than 3 months after resection, 2 weeks after completing chemoradiation therapy. He was found to have a dissecting right vertebral artery aneurysm and underwent coil sacrifice after ventriculostomy placement. The external ventricular drain (EVD) was ultimately converted to a shunt, and he was discharged at the baseline neurological condition. However, during the next 2 months, the patient had aggressive tumor growth requiring multiple shunt revisions due to highly proteinaceous CSF causing proximal shunt failure. His family ultimately chose to withdraw care due to shunt- and hydrocephalusrelated complications. The third patient was a 9-year-old female who had a history of a myelomeningocele, Chiari II malformation, and shunted hydrocephalus who presented with a right ACA aneurysm adjacent to the shunt catheter tip, raising the possibility of a traumatic etiology. She underwent uneventful clipping and was discharged at her neurological baseline, with an mRS score of 4 . The last patient was a 14-year-old female who presented with a Hunt and Hess grade IV SAH from a ruptured giant, left middle cerebral artery (MCA) aneurysm. She underwent successful clipping, and had improving residual hemiparesis with the ability to ambulate independently at the last follow-up, with an mRS score of 3.

Patients were monitored with MRI and MRA. No de novo aneurysms were found in our series on follow-up im- 
TABLE 2. Summary of patient characteristics of all pediatric intracranial aneurysms 1991-2004 and 2004-2021, and overall 1991-2021

\begin{tabular}{lccc}
\hline & $1991-2004$ & $2004-2021$ & $1991-2021$ \\
\hline No. of pts & 19 & 28 & 47 \\
\hline Incidence per yr & 1.4 & 1.6 & 1.5 \\
\hline Mean age, yrs & $9.8 \pm 5.7$ & $12.7 \pm 5.2$ & $11.5 \pm 5.4$ \\
\hline No. of aneurysms & 19 & 34 & 53 \\
\hline Male sex & $13(68.4)$ & $16(57.1)$ & $29(61.7)$ \\
\hline SAH & $11(57.9)$ & $14(50.0)$ & $25(53.2)$ \\
\hline Infectious & $1(5.3)$ & $0(0)$ & $1(2.1)$ \\
\hline Anterior location & $11(57.9)$ & $28(82.4)$ & $39(73.6)$ \\
\hline Giant & $7(36.8)$ & $4(11.8)$ & $11(20.8)$ \\
\hline Treatment & & & \\
\hline \multicolumn{1}{c}{ Surgical } & $13(68.4)$ & $18(52.9)$ & $31(58.5)$ \\
\hline Endovascular & $3(15.8)$ & $12(35.3)$ & $15(28.3)$ \\
\hline Antibiotics & $1(5.3)$ & $0(0)$ & $1(1.9)$ \\
\hline $\begin{array}{l}\text { Neurological outcomes } \\
\text { (treated pts) }\end{array}$ & & & \\
\hline \multicolumn{1}{c}{ mRS score $\leq 2$} & $15(83.3)$ & $25(96.2)$ & $40(90.9)$ \\
\hline Mortality & $0(0)$ & $2(7.7)$ & $2(4.5)$ \\
\hline $\begin{array}{l}\text { Overall mortality } \\
\text { Values represent the number of patients (\%), number of aneurysms (\%), or } \\
\text { mean } \pm \text { SD unless indicated otherwise. }\end{array}$ & &
\end{tabular}

aging over a mean follow-up period of 65.9 months (5.5 years). Only 1 patient experienced aneurysm regrowth after treatment, and this was a 1-year-old patient who had a left MCA infarct during resection of a left-sided, desmoplastic infantile ganglioma at an outside institution. She experienced a subdural, intraparenchymal, and subarachnoid hemorrhage 17 days after surgery, and was found to have a left MCA pseudoaneurysm, presumably due to MCA injury during index tumor resection. She was transferred to our institution, and the pseudoaneurysm re-ruptured on arrival. The patient underwent an emergency left hemicraniectomy and EVD placement, and the pseudoaneurysm was coiled with dome occlusion. The patient was stabilized, extubated, and ultimately discharged from the hospital. Four months later, she was admitted electively for Pipeline-assisted coiling of the MCA pseudoaneurysm. Intraoperatively, the aneurysm was noted to have increased in size from $5 \mathrm{~mm}$ to $40 \mathrm{~mm}$. The aneurysm was successfully coiled and flow diverted. One year later, the patient was moving all extremities with subtle right-sided weakness without evidence of aneurysm recurrence.

\section{Discussion}

Pediatric intracranial aneurysms are a rare entity. We previously reported a series of 19 pediatric patients treated at our institution from 1991 to 2004, representing an aneurysm presentation rate of approximately 1.4 patients per year at a large academic institution. ${ }^{21}$ Here, we present our institutional experience from 1991 to 2021, including 47 pediatric patients with 53 aneurysms during this time pe-
TABLE 3. Pediatric versus adult aneurysm characteristics from patients treated at our institution, 1991-2021

\begin{tabular}{|c|c|c|c|}
\hline & Pediatric (\%) & Adult (\%) & $\mathrm{p}$ Value \\
\hline $\operatorname{Sex}^{*}$ & & & $<0.001$ \\
\hline M & $29(61.7)$ & $1024(23.0)$ & \\
\hline $\mathrm{F}$ & $18(38.3)$ & $3429(77.0)$ & \\
\hline $\mathrm{SAH}^{*}$ & $25(53.2)$ & $1620(36.4)$ & 0.026 \\
\hline Location† & & & 0.771 \\
\hline Anterior & $39(73.6)$ & $3609(70.8)$ & \\
\hline Posterior & $14(26.4)$ & $1488(29.2)$ & \\
\hline Site† & & & $<0.001$ \\
\hline Cavernous ICA & $5(9.6)$ & $138(2.7)$ & 0.011 \\
\hline Ophthalmic ICA & $2(3.8)$ & $596(11.7)$ & 0.115 \\
\hline Other ICA & $3(5.7)$ & $692(13.6)$ & 0.140 \\
\hline AChA & $0(0)$ & $70(1.4)$ & 0.793 \\
\hline ICA termination & $6(11.3)$ & $38(0.7)$ & $<0.001$ \\
\hline ACA & $9(17.0)$ & $187(3.7)$ & $<0.001$ \\
\hline $\mathrm{ACoA}$ & $3(5.7)$ & $1017(20.0)$ & 0.015 \\
\hline MCA & $11(20.8)$ & 871 (17.1) & 0.602 \\
\hline Basilar & $2(3.8)$ & $312(6.1)$ & 0.673 \\
\hline AICA & $0(0)$ & $20(0.4)$ & $>0.99$ \\
\hline PICA & $1(1.9)$ & $121(2.4)$ & $>0.99$ \\
\hline Vertebral & $3(5.7)$ & $58(1.1)$ & 0.017 \\
\hline Vertebrobasilar junction & $0(0)$ & $33(0.6)$ & $>0.99$ \\
\hline $\mathrm{PCoA}$ & $3(5.7)$ & $813(16.0)$ & 0.064 \\
\hline PCA & $4(7.5)$ & $53(1.0)$ & $<0.001$ \\
\hline SCA & $1(1.9)$ & $78(1.5)$ & $>0.99$ \\
\hline Size $\geq 25 \mathrm{~mm} \dagger$ & $10(18.9)$ & $119(2.3)$ & $<0.001$ \\
\hline
\end{tabular}

$\mathrm{AChA}=$ anterior choroidal artery; AICA = anterior inferior cerebellar artery; $\mathrm{PICA}=$ posterior inferior cerebellar artery; $\mathrm{SCA}=$ superior cerebellar artery.

* Presented as the number of patients (\%).

$\dagger$ Presented as the number of aneurysms (\%).

Boldface type indicates statistical significance.

riod, corresponding to an overall incidence of 1.5 patients per year. Remarkably, this incidence of approximately 1.4 to 1.6 patients per year has held relatively constant over 3 decades. During this time, pediatric aneurysms represented $1 \%$ of all 5150 aneurysms treated at our institution.

There is a well-established male dominance for pediatric aneurysms but a female dominance for cerebral aneurysms that present during adulthood. ${ }^{3,20,23-28}$ The mechanisms underlying this clinical observation remain unclear. Recently, Bakker et al. published a large genome-wide association study of intracranial aneurysms, identifying 17 risk loci from 10,754 cases and 306,882 controls of European and East Asian ancestry. ${ }^{29}$ As anticipated, the study revealed a polygenic architecture and no substantially different genetics between ruptured and unruptured intracranial aneurysms. This study did not find clear sex-specific genetic markers associated with intracranial aneurysms. Whether these sex-specific observations are a function of transient pubertal changes or sex-associated behavioral or environmental factors remains to be elucidated.

The distinctly different epidemiology of pediatric and 
TABLE 4. Summary of pediatric versus adult aneurysm characteristics from patients treated at our institution, 1991-2021

\begin{tabular}{lcc}
\hline & Pediatric & Adult \\
\hline Pt sex & M predominance & F predominance \\
\hline Pts presenting & $53 \%(\sim 1$ in 2$)$ & $37 \%(\sim 1$ in 3) \\
w/ SAH & & \\
\hline Aneurysm & More likely in cavernous & More likely in ACoA \\
location & ICA, ICA termination, & \\
& ACA, VAs, \& PCA & \\
\hline Aneurysm size & $21 \%(\sim 1$ in 5$) \geq 25 \mathrm{~mm}$ & $2 \%(\sim 1$ in 50$) \geq 25 \mathrm{~mm}$ \\
\hline
\end{tabular}

adult aneurysm cases suggests different etiologies for these lesions (Table 4). Adult aneurysms are thought to originate from missing patches of tunica media that, with time and under the influence of specific environmental factors, give rise to fully developed aneurysms by the 6th and 7 th decades of life..$^{30}$ In contrast, pediatric aneurysms are probably almost fully developed lesions at the time of birth and are therefore better considered congenital vascular abnormalities. This may account for the markedly greater proportion of giant $(\geq 25 \mathrm{~mm})$ pediatric aneurysms $(20.8 \%$ vs $2.3 \%)$ and their increased propensity to present with rupture (53.2\% vs $36.4 \%$ ), which is highly suggestive of a congenitally thin aneurysm wall. Furthermore, there is a higher male prevalence of pediatric aneurysms $(61.7 \%$ vs $23.0 \%$ ), which is in keeping with the greater proportion of males with other congenital vascular lesions, such as transposition of the great vessels, tetralogy of Fallot, left ventricular hypoplasia syndrome, and aortic coarctation..$^{31}$

Compared with adult aneurysms at our institution, pediatric aneurysms were more likely to present with an SAH (53.2\% vs $36.4 \%, \mathrm{p}=0.026)$. Our findings are commensurate with those of Beez et al., who summarized 573 patients with 656 aneurysms, from 2000 to 2015, and found that $55 \%$ of patients presented with an SAH. ${ }^{32}$ Similar to other studies, our experience also demonstrates that a substantially higher proportion of pediatric aneurysms are giant, $20.8 \%$, versus $2.3 \%$ in adults $(\mathrm{p}<0.001)$. Although pediatric aneurysms were overall similar in distribution compared with their adult counterparts in an anterior versus posterior circulation location, their specific vascular distribution differed significantly (Table 3 ). Pediatric aneurysms occurred predominantly at the MCA bifurcation and were statistically more likely to occur in the ICA termination, ACA, cavernous ICA, vertebral artery, and PCA compared with adult aneurysms. Notably, adult aneurysms were much more likely to occur within the anterior communicating artery compared with pediatric aneurysms. Interestingly, while only one pediatric patient had an intranidal aneurysm associated with an arteriovenous malformation (AVM), interrogation of our adult cohort of patients with AVMs revealed 25 of 482 patients (5.2\%) with an intranidal AVM. Therefore, the pediatric rate was statistically similar to that of the adult patient population $(\mathrm{p}=0.72)$.

Overall, $85.1 \%$ of patients in our experience had good neurological outcomes with an $\mathrm{mRS}$ score $\leq 2$ at the last follow-up. Excluding the 3 patients who were neurologi-
TABLE 5. Treatment of pediatric cerebral aneurysms by endovascular versus open techniques over time

\begin{tabular}{cccr}
\hline Decade & $\begin{array}{c}\text { No. of Treated } \\
\text { Aneurysms }\end{array}$ & $\begin{array}{c}\text { Endovascular } \\
\text { Treatment }\end{array}$ & $\begin{array}{c}\text { Surgical } \\
\text { Treatment }\end{array}$ \\
\hline $1991-2001$ & 8 & $2(25.0)$ & $6(75.0)$ \\
\hline $2002-2011$ & 14 & $5(35.7)$ & $9(64.3)$ \\
\hline $2012-2021$ & 24 & $8(33.3)$ & $16(66.7)$ \\
\hline
\end{tabular}

cally devastated and lost brainstem reflexes shortly after arrival to the hospital and were not treated, $90.9 \%$ of treated patients achieved good neurological outcome. Of the remaining patients, 1 patient died due to complications from anaplastic astrocytoma progression, and 1 patient was not able to walk preoperatively due to congenital myelomeningocele. Thus, although the overall mortality rate in our series was 5 of 47 patients (10.6\%), only 1 patient $(2.3 \%$, $1 / 44$ ) who was treated died due to SAH-related complications. Similarly, only 1 patient $(2.3 \%, 1 / 44)$ had residual neurological deficits requiring assistance during activities of daily living, which was attributable to the cerebral aneurysm. These results demonstrate that despite more than $50 \%$ of patients presenting with an SAH, excellent neurological outcomes are still achievable.

Our clinical practice in treating pediatric intracranial aneurysms reflects the rise in endovascular techniques over the past 30 years. ${ }^{4,12,15,16,19,28,33}$ While only 2 aneurysms $(25.0 \%)$ were treated endovascularly between 1991 and 2001 at our institution, 5 aneurysms (35.7\%) were treated endovascularly between 2002 and 2011, and 8 (33.3\%) were treated endovascularly in the next decade (Table 5). As an institution, we were relatively early adopters of endovascular technology when we successfully treated a giant, left cavernous ICA pediatric aneurysm with balloon occlusion in 1994. In the 1st decade, both cases of endovascularly treated patients were balloon occlusions of giant, cavernous ICA aneurysms. From 2002 to 2011, a combination of balloon occlusion, coiling, and glue embolization was used. Beginning in 2012, select patients were treated with flow diversion in addition to coiling. The longterm outcomes for these newer endovascular techniques over a patient's lifetime, however, are unproven and must be studied carefully.

A key difference in adult versus pediatric aneurysms, however, is that pediatric patients have potentially 70 or more years of life expectancy. Thus, treatment goals for cerebral aneurysms should ideally be curative-not simply mitigating hemorrhage risk. Despite the significant advancements that have been made in endovascular techniques, the longevity of endovascular solutions remains in question, as multiple trials have demonstrated higher retreatment rates of aneurysms treated endovascularly. ${ }^{34,35}$ In line with these observations, no patient who underwent microsurgical clipping had aneurysm recurrence. In our practice, permanent endovascular treatments of large, cavernous segment ICA aneurysms and/or vertebral artery aneurysms were preceded by balloon test occlusion followed by vessel sacrifice when able. No patient who underwent parent-vessel sacrifice had recurrence of their aneurysm. 
TABLE 6. Summary of major pediatric intracranial aneurysm series since 2005

\begin{tabular}{|c|c|c|c|c|c|}
\hline Authors \& Year & No. of Pts & SAH (\%) & Surgical Treatment & Endovascular Treatment & Mortality \\
\hline Huang et al., $2005^{21}$ & 19 & $11(57.9)$ & $13(81.3)$ & $3(18.8)$ & $1(5.3)$ \\
\hline Agid et al., $2005^{33}$ & 33 & $9(27.3)$ & $10(43.5)$ & $13(56.5)$ & $5(15.2)$ \\
\hline Lasjaunias et al., $2005^{40}$ & 59 & $27(45.8)$ & $10(31.3)$ & $25(78.1)$ & $5(8.5)$ \\
\hline Sanai et al., $2006^{27}$ & 32 & 7 (21.9) & $13(44.8)$ & $16(55.2)$ & $0(0)$ \\
\hline Sharma et al., $2007^{43}$ & 55 & 43 (78.2) & $40(90.9)$ & $4(9.1)$ & $5(9.1)$ \\
\hline Stiefel et al., $2008^{19}$ & 12 & $12(100)$ & $8(61.5)$ & $5(38.5)$ & $1(8.3)$ \\
\hline Vaid et al., $2008^{20}$ & 27 & $23(85.2)$ & $24(100)$ & $0(0)$ & $3(11.1)$ \\
\hline Hetts et al., $2009^{25}$ & 77 & $25(32.5)$ & $29(49.2)$ & $30(50.8)$ & $1(1.3)$ \\
\hline Liang et al., $2009^{11}$ & 24 & $11(45.8)$ & $4(21.1)$ & $15(78.9)$ & $1(4.2)$ \\
\hline Lv et al., $2009^{12}$ & 25 & $11(44)$ & $0(0)$ & $25(100)$ & $1(4)$ \\
\hline Kakarla et al., $2010^{39}$ & 48 & $13(27.1)$ & $72(100)$ & $0(0)$ & $1(2.1)$ \\
\hline Requejo et al., $2010^{42}$ & 17 & $4(23.5)$ & $4(28.6)$ & $10(71.4)$ & $3(17.6)$ \\
\hline Fulkerson et al., $2011^{7}$ & 28 & $19(67.9)$ & $18(69.2)$ & $10(38.5)$ & $2(7.1)$ \\
\hline Saleh \& Dawson, $2011^{15}$ & 12 & Unreported & $0(0)$ & $12(100)$ & $1(8.3)$ \\
\hline Koroknay-Pál et al., $2012^{26}$ & 114 & $89(78.1)$ & $80(96.4)$ & $3(3.6)$ & $40(35.1)$ \\
\hline Saraf et al., $2012^{16}$ & 23 & $13(56.5)$ & $0(0)$ & $23(100)$ & $0(0)$ \\
\hline Mehrotra et al., $2012^{41}$ & 57 & $50(87.7)$ & $57(100)$ & $0(0)$ & $5(8.8)$ \\
\hline Garg et al., $2014^{37}$ & 62 & $36(58.1)$ & $36(65.5)$ & $19(34.5)$ & $1(1.6)$ \\
\hline Takemoto et al., $2014^{28}$ & 31 & $15(48.4)$ & $0(0)$ & $34(100)$ & $0(0)$ \\
\hline Gross et al., $2015^{38}$ & 33 & $8(24.2)$ & $8(34.8)$ & $15(65.2)$ & $0(0)$ \\
\hline Deora et al., $2017^{36}$ & 44 & $35(79.5)$ & $40(100)$ & $0(0)$ & $1(2.3)$ \\
\hline Chen et al., $2018^{23}$ & 64 & $31(48.8)$ & $34(54.8)$ & $28(45.2)$ & $1(1.6)$ \\
\hline Yasin et al., $2019^{44}$ & 42 & $15(26.3)$ & $13(34.2)$ & $25(65.8)$ & $2(4.8)$ \\
\hline Kim et al., $2019^{9}$ & 26 & $16(61.5)$ & $10(50)$ & $10(50)$ & $1(3.9)$ \\
\hline Nam et al., $2019^{13}$ & 23 & $11(47.8)$ & $3(13.6)$ & $20(90.9)$ & $1(4.3)$ \\
\hline Slator et al., $2019^{17}$ & 22 & $22(100)$ & $4(20)$ & $16(80)$ & $4(18.2)$ \\
\hline Amelot et al., $2019^{45}$ & 51 & $37(72.5)$ & $9(17.6)$ & $42(82.4)$ & $10(19.6)$ \\
\hline Cherian et al., $2020^{24}$ & 39 & $5(12.8)$ & $0(0)$ & $46(100)$ & $3(7.7)$ \\
\hline
\end{tabular}

Percentages are based on the number of treated patients.

The only case of mortality related to rehemorrhage in our series occurred while treating bilateral enlarging ICA aneurysms endovascularly via flow diversion in one ICA and coiling in the other. Similarly, the only case of aneurysm recurrence occurred during treatment of a ruptured, left MCA pseudoaneurysm, where coil embolization only achieved dome protection initially. The patient was neurologically stabilized and brought back electively for definitive treatment with additional coils and flow diversion.

Since our last description of 19 patients with cerebral aneurysms in 2005,28 publications have reported outcomes on the treatment of pediatric intracranial aneurysms

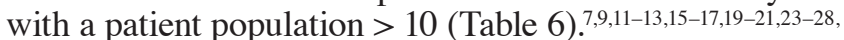
33,36-45 Remarkably, methods for the treatment of pediatric aneurysms have ranged from $100 \%$ microsurgical treatment to $100 \%$ endovascular technologies, with ruptured presentations ranging from $13 \%$ to more than $80 \%$. Cherian et al. described the treatment of 39 patients with 50 intracranial aneurysms, 5 of whom were treated after acute SAH with flow diversion. ${ }^{24}$ Two $(40 \%)$ of these 5 patients experienced perioperative rehemorrhage and died. Amelot et al. published a series of 51 patients, $72.5 \%$ of whom pre- sented with an SAH; $;{ }^{45} 82.4 \%$ were treated endovascularly, and they reported a morality rate of $19.6 \%$. Conversely, Kakarla et al. described outcomes in 48 patients wherein $100 \%$ were treated with microsurgery. ${ }^{39}$ Despite the use of bypass techniques in $17 \%$ of patients as well as the use of hypothermic circulatory arrest, 3 patients required endovascular salvage procedures to treat residual/recurrent aneurysms, and 1 patient died 13 months later, likely due to rehemorrhage. These results demonstrate that although microsurgical and endovascular techniques are both able to treat pediatric intracranial aneurysms with good results, neither is sufficient to treat all aneurysmal pathology. In our practice, the treatment of each pediatric intracranial aneurysm is guided by a team of cerebrovascular and endovascular neurosurgeons, as well as neurointerventional radiologists. The risks, benefits, and likely long-term outcomes are discussed before a collaborative decision is made with the family. Ultimately, our series reflects the nuanced practice of a large academic institution without a significant operative bias.

Limitations to our study include its retrospective analysis of a prospectively collected cohort of patients. We 
acknowledge that there may be a referral bias to a large tertiary academic institution. For example, we only experienced 1 of 47 patients with a mycotic aneurysm, which is low compared with other series reporting rates ranging from $3 \%$ to $20 \%$. While neighboring children's hospitals perform cerebral angiograms and initial medical workup, generally our hospital receives transfers due to a need for microsurgical or endovascular interventions. Our mean length of follow-up was 65.9 months, although every attempt was made to recontact family members through available patient records. We did not discover de novo aneurysms with MRA, although this may underestimate the true incidence of small preaneurysm changes, as MRA is limited by susceptibility artifact. ${ }^{6}$ Nonetheless, although the gold standard for imaging of cerebral aneurysms continues to be DSA, follow-up of pediatric patients over the course of a lifetime with angiography is limited by its invasiveness and additional radiation dosage.

\section{Conclusions}

Pediatric intracranial aneurysms are rare and complex lesions that require nuanced open microsurgical and endovascular analysis to optimize long-term outcomes. Over the course of 30 years, we encountered approximately 1.5 patients per year, with half presenting with an SAH. Male predominance, location prevalence, and size differences between pediatric and adult aneurysms suggest distinct pathophysiological and potentially genetic mechanisms, although future studies are necessary to corroborate these clinical observations. Excluding patients who were unable to regain brainstem reflexes, the mortality rate was $2.3 \%$, and the aneurysm recurrence rate was $2.3 \%$. Among 44 of the 47 pediatric patients who were treated, $90.9 \%$ had an $\mathrm{mRS}$ score $\leq 2$ at the last follow-up. No aneurysms recurred after microsurgical clipping. Our only case of rebleed was in a patient with multiple aneurysms who bled while awaiting treatment of a second aneurysm as an inpatient. Further lifelong follow-up is required to truly ascertain aneurysm recurrence or rebleed risk for pediatric intracranial aneurysms.

\section{References}

1. Krishna H, Wani AA, Behari S, Banerji D, Chhabra DK, Jain VK. Intracranial aneurysms in patients 18 years of age or under, are they different from aneurysms in adult population? Acta Neurochir (Wien). 2005;147(5):469-476.

2. Vasan R, Patel J, Sweeney JM, Carpenter AM, Downes K, Youssef AS, et al. Pediatric intracranial aneurysms: current national trends in patient management and treatment. Childs Nerv Syst. 2013;29(3):451-456.

3. Alawi A, Edgell RC, Elbabaa SK, Callison RC, Khalili YA, Allam H, Alshekhlee A. Treatment of cerebral aneurysms in children: analysis of the Kids' Inpatient Database. J Neurosurg Pediatr. 2014;14(1):23-30.

4. Bhogal P, Pérez MA, Wendl C, Bäzner H, Ganslandt O, Henkes H. Paediatric aneurysms - review of endovascular treatment strategies. J Clin Neurosci. 2017;45:54-59.

5. Colby GP, Jiang B, Bender MT, Beaty NB, Westbroek EM, $\mathrm{Xu}$ R, et al. Pipeline-assisted coil embolization of a large middle cerebral artery pseudoaneurysm in a 9-month-old infant: experience from the youngest flow diversion case. $J$ Neurosurg Pediatr. 2018;22(5):532-540.
6. Deutschmann HA, Augustin M, Simbrunner J, Unger B, Schoellnast H, Fritz GA, Klein GE. Diagnostic accuracy of 3D time-of-flight MR angiography compared with digital subtraction angiography for follow-up of coiled intracranial aneurysms: influence of aneurysm size. AJNR Am J Neuroradiol. 2007;28(4):628-634.

7. Fulkerson DH, Voorhies JM, Payner TD, Leipzig TJ, Horner TG, Redelman K, Cohen-Gadol AA. Middle cerebral artery aneurysms in children: case series and review. J Neurosurg Pediatr. 2011:8(1):79-89.

8. Jian BJ, Hetts SW, Lawton MT, Gupta N. Pediatric intracranial aneurysms. Neurosurg Clin N Am. 2010;21(3):491-501.

9. Kim M, Lee HS, Lee S, Park JC, Ahn JS, Kwon DH, et al. Pediatric intracranial aneurysms: favorable outcomes despite rareness and complexity. World Neurosurg. 2019;125:e1203e1216.

10. Krings T, Geibprasert S, terBrugge KG. Pathomechanisms and treatment of pediatric aneurysms. Childs Nerv Syst. 2010;26(10):1309-1318.

11. Liang J, Bao Y, Zhang H, Wrede KH, Zhi X, Li M, Ling F. The clinical features and treatment of pediatric intracranial aneurysm. Childs Nerv Syst. 2009;25(3):317-324.

12. Lv X, Jiang C, Li Y, Yang X, Wu Z. Endovascular treatment for pediatric intracranial aneurysms. Neuroradiology. 2009; 51(11):749-754.

13. Nam SM, Jang D, Wang KC, Kim SK, Phi JH, Lee JY, et al. Characteristics and treatment outcome of intracranial aneurysms in children and adolescents. J Korean Neurosurg Soc. 2019;62(5):551-560.

14. Navarro R, Brown BL, Beier A, Ranalli N, Aldana P, Hanel RA. Flow diversion for complex intracranial aneurysms in young children. J Neurosurg Pediatr. 2015;15(3):276-281.

15. Saleh E, Dawson RC III. Endovascular management of pediatric aneurysms. Neuroradiol J. 2011;24(5):693-698.

16. Saraf R, Shrivastava M, Siddhartha W, Limaye U. Intracranial pediatric aneurysms: endovascular treatment and its outcome. J Neurosurg Pediatr. 2012;10(3):230-240.

17. Slator N, Talibi SS, Mundil N, Thomas A, Lamin S, Walsh R, et al. Paediatric intracranial aneurysms: a British institutional review. Childs Nerv Syst. 2019;35(7):1197-1205.

18. Sorteberg A, Dahlberg D. Intracranial non-traumatic aneurysms in children and adolescents. Curr Pediatr Rev. 2013; 9(4):343-352.

19. Stiefel MF, Heuer GG, Basil AK, Weigele JB, Sutton LN, Hurst RW, Storm PB. Endovascular and surgical treatment of ruptured cerebral aneurysms in pediatric patients. Neurosurgery. 2008;63(5):859-866.

20. Vaid VK, Kumar R, Kalra SK, Mahapatra AK, Jain VK. Pediatric intracranial aneurysms: an institutional experience. Pediatr Neurosurg. 2008;44(4):296-301.

21. Huang J, McGirt MJ, Gailloud P, Tamargo RJ. Intracranial aneurysms in the pediatric population: case series and literature review. Surg Neurol. 2005;63(5):424-433.

22. International Study of Unruptured Intracranial Aneurysms Investigators. Unruptured intracranial aneurysms - risk of rupture and risks of surgical intervention. $N$ Engl J Med. 1998;339(24):1725-1733.

23. Chen R, Zhang S, You C, Guo R, Ma L. Pediatric intracranial aneurysms: changes from previous studies. Childs Nerv Syst. 2018;34(9):1697-1704.

24. Cherian J, Srinivasan V, Froehler MT, Grossberg JA, Cawley CM, Hanel RA, et al. Flow diversion for treatment of intracranial aneurysms in pediatric patients: multicenter case series. Neurosurgery. 2020;87(1):53-62.

25. Hetts SW, Narvid J, Sanai N, Lawton MT, Gupta N, Fullerton HJ, et al. Intracranial aneurysms in childhood: 27-year single-institution experience. AJNR Am J Neuroradiol. 2009; 30(7):1315-1324.

26. Koroknay-Pál P, Lehto H, Niemelä M, Kivisaari R, Hernesni- 
emi J. Long-term outcome of 114 children with cerebral aneurysms. J Neurosurg Pediatr. 2012;9(6):636-645.

27. Sanai N, Quinones-Hinojosa A, Gupta NM, Perry V, Sun PP, Wilson CB, Lawton MT. Pediatric intracranial aneurysms: durability of treatment following microsurgical and endovascular management. J Neurosurg. 2006;104(2)(suppl):82-89.

28. Takemoto K, Tateshima S, Golshan A, Gonzalez N, Jahan R, Duckwiler G, Vinuela F. Endovascular treatment of pediatric intracranial aneurysms: a retrospective study of 35 aneurysms. J Neurointerv Surg. 2014;6(6):432-438.

29. Bakker MK, van der Spek RAA, van Rheenen W, Morel S, Bourcier R, Hostettler IC, et al. Genome-wide association study of intracranial aneurysms identifies 17 risk loci and genetic overlap with clinical risk factors. Nat Genet. 2020; 52(12):1303-1313.

30. Carmichael R. The pathogenesis of noninflammatory cerebral aneurysms. J Pathol Bacteriol. 1950;62(1):1-19.

31. Abeywardana S, Sullivan EA. Congenital Anomalies in Australia 2002-2003. Australian Institute of Health and Welfare National Perinatal Statistics Unit; 2008.

32. Beez T, Steiger HJ, Hänggi D. Evolution of management of intracranial aneurysms in children: a systematic review of the modern literature. J Child Neurol. 2016;31(6):773-783.

33. Agid R, Souza MP, Reintamm G, Armstrong D, Dirks P, TerBrugge KG. The role of endovascular treatment for pediatric aneurysms. Childs Nerv Syst. 2005;21(12):1030-1036.

34. Darsaut TE, Jack AS, Kerr RS, Raymond J. International Subarachnoid Aneurysm Trial-ISAT part II: study protocol for a randomized controlled trial. Trials. 2013;14:156.

35. Spetzler RF, McDougall CG, Zabramski JM, Albuquerque FC, Hills NK, Nakaji P, et al. Ten-year analysis of saccular aneurysms in the Barrow Ruptured Aneurysm Trial. J Neurosurg. 2019;132(3):771-776.

36. Deora H, Rao KVLN, Somanna S, Srinivas D, Shukla DP, Bhat DI. Surgically managed pediatric intracranial aneurysms: how different are they from adult intracranial aneurysms? Pediatr Neurosurg. 2017;52(5):313-317.

37. Garg K, Singh PK, Sharma BS, Chandra PS, Suri A, Singh $\mathrm{M}$, et al. Pediatric intracranial aneurysms-our experience and review of literature. Childs Nerv Syst. 2014;30(5):873883.

38. Gross BA, Smith ER, Scott RM, Orbach DB. Intracranial aneurysms in the youngest patients: characteristics and treatment challenges. Pediatr Neurosurg. 2015;50(1):18-25.

39. Kakarla UK, Beres EJ, Ponce FA, Chang SW, Deshmukh VR, Bambakidis NC, et al. Microsurgical treatment of pediatric intracranial aneurysms: long-term angiographic and clinical outcomes. Neurosurgery. 2010;67(2):237-250.
40. Lasjaunias P, Wuppalapati S, Alvarez H, Rodesch G, Ozanne A. Intracranial aneurysms in children aged under 15 years: review of 59 consecutive children with 75 aneurysms. Childs Nerv Syst. 2005;21(6):437-450

41. Mehrotra A, Nair AP, Das KK, Srivastava A, Sahu RN, Kumar R. Clinical and radiological profiles and outcomes in pediatric patients with intracranial aneurysms. J Neurosurg Pediatr. 2012;10(4):340-346.

42. Requejo F, Ceciliano A, Cardenas R, Villasante F, Jaimovich R, Zuccaro G. Cerebral aneurysms in children: are we talking about a single pathological entity? Childs Nerv Syst. 2010;26(10):1329-1335.

43. Sharma BS, Sinha S, Mehta VS, Suri A, Gupta A, Mahapatra AK. Pediatric intracranial aneurysms-clinical characteristics and outcome of surgical treatment. Childs Nerv Syst. 2007;23(3):327-333.

44. Yasin JT, Wallace AN, Madaelil TP, Osbun JW, Moran CJ, Cross DT, et al. Treatment of pediatric intracranial aneurysms: case series and meta-analysis. J Neurointerv Surg. 2019;11(3):257-264.

45. Amelot A, Saliou G, Benichi S, Alias Q, Boulouis G, Zerah $\mathrm{M}$, et al. Long-term outcomes of cerebral aneurysms in children. Pediatrics. 2019;143(6):e20183036.

\section{Disclosures}

Christopher M. Jackson: scientific founder and CMO of Egret Therapeutics.

\section{Author Contributions}

Conception and design: Tamargo, $\mathrm{Xu}$. Acquisition of data: Tamargo, Xu, Yang, Gailloud, Caplan, C Jackson, EM Jackson, Groves, Robinson, Cohen, Huang. Analysis and interpretation of data: Xu, Xie. Drafting the article: Tamargo, Xu, Xie. Critically revising the article: all authors. Reviewed submitted version of manuscript: all authors. Approved the final version of the manuscript on behalf of all authors: Tamargo. Statistical analysis: $\mathrm{Xu}, \mathrm{Xie}$. Administrative/technical/material support: Tamargo. Study supervision: Tamargo.

\section{Correspondence}

Rafael J. Tamargo: Johns Hopkins University School of Medicine, Johns Hopkins Hospital, Baltimore, MD. rtamarg@jhmi.edu. 